\title{
PAM-6 Coded Modulation for IM/DD Channels with a Peak-Power Constraint
}

\author{
Tobias Prinz*, Thomas Wiegart*, Daniel Plabst*, \\ Stefano Calabrò ${ }^{\dagger}$, Georg Böcherer ${ }^{\dagger}$, Nebojsa Stojanovic ${ }^{\dagger}$, Talha Rahman ${ }^{\dagger}$ \\ * Institute for Communications Engineering, Technical University of Munich \\ ${ }^{\dagger}$ German Research Center, Huawei Technologies Duesseldorf GmbH \\ Email: \{tobias.prinz, thomas.wiegart, d.plabst\}@tum.de \\ \{stefano.calabro, georg.bocherer, nebojsa.stojanovic, talha.rahman $\} @$ huawei.com
}

\begin{abstract}
Coded modulation with probabilistic amplitude shaping (PAS) is considered for intensity modulation/direct detection channels with a transmitter peak-power constraint. PAS is used to map bits to a uniform PAM-6 distribution and outperforms PAM-8 for rates up to around 2.3 bits per channel use. PAM-6 with PAS also outperforms a cross-shaped QAM-32 constellation by up to $1 \mathrm{~dB}$ and $0.65 \mathrm{~dB}$ after bit-metric soft- and hard decoding, respectively. An alternative PAM-6 scheme based on a framed-cross-shaped QAM-32 constellation is proposed that shows similar gains.
\end{abstract}

\section{INTRODUCTION}

Short-reach optical links are often based on transceivers that use intensity modulation (IM) and direct detection (DD). This allows for cheap hardware, low power consumption, and low latency [1], [2]. Since short-reach systems are usually operated without optical amplifiers, practical constraints at the transmitter laser and modulator dictate a peak power constraint [3].

Early IM/DD systems were based on on-off keying (OOK), while newer systems use unipolar 4-ary and 8-ary pulse amplitude modulation (PAM) formats to increase data rates. The higher-order modulation comes at the price of increased complexity, especially at the receiver. The number of symbols in a modulation alphabet is usually a power of two to allow for an easy integration with binary data and binary forward error correction (FEC). However, under a peak power constraint, other modulation alphabets give larger information rates, especially for small alphabet sizes.

We study three schemes to transmit PAM-6. First, we review the benchmark cross-shaped QAM-32 constellation shown in Fig. 3 below [4], [5]. Second, we propose a modified QAM-32 constellation with a framed-cross shape which improves information rates. Third, we use probabilistic amplitude shaping (PAS) [6] to map bits to uniformly-spaced signal constellations whose number of points $M$ is even but not a power of two. The idea is that a distribution matcher (DM) creates a block of PAM- $(M / 2)$ symbols that are combined with parity bits from a FEC code to obtain a block of PAM- $M$ symbols. Of course, PAS algorithms can also put out non-uniform distributions.

\section{PREliminaries}

\section{A. System Model}

Consider a peak-power constrained additive white Gaussian noise (AWGN) channel with output

$$
Y=X+N
$$

where $X$ is the transmitted signal, and $N$ is AWGN with $N \sim \mathcal{N}\left(0, \sigma^{2}\right)$. We consider the PAM- $M$ alphabet

$$
\mathcal{X}=\left\{0, \frac{\sqrt{P_{\max }}}{M-1}, \ldots,(M-1) \frac{\sqrt{P_{\max }}}{M-1}\right\}
$$

where $P_{\max }$ is the peak transmit power. The peak-signal-tonoise-power ratio is defined as

$$
\operatorname{PSNR}=\frac{P_{\max }}{\sigma^{2}} .
$$

\section{B. Spectral Efficiencies}

We study the spectral efficiencies (SEs) $R_{S M D}, R_{B M D}$, $\mathrm{R}_{\mathrm{HD}, \mathrm{SMD}}$ and $\mathrm{R}_{\mathrm{HD}, \mathrm{BMD}}$ under symbol metric decoding (SMD) and bit metric decoding (BMD), and under soft decoding (SD) and hard decoding (HD), respectively. We refer to [7, Eq. (1)] and [8, Chap. 8] for more details. Unless stated otherwise, we use uniformly distributed input symbols with probability $P_{X}(x)=1 / M, \forall x \in \mathcal{X}$.

The SE for SD-SMD is

$$
\mathrm{R}_{\mathrm{SMD}}=\mathrm{I}(X ; Y)=\mathrm{H}(X)-\mathrm{H}(X \mid Y)
$$

where $\mathrm{I}(\cdot ; \cdot)$ denotes mutual information, and $\mathrm{H}(\cdot)$ and $\mathrm{H}(\cdot \mid \cdot)$ denote entropy and conditional entropy, respectively.

For BMD, we represent each symbol random variable (RV) $X$ by a sequence of binary RVs $\left(B_{1}, \ldots, B_{m}\right)$, where $m=\left\lceil\log _{2} M\right\rceil$. The SE for SD-BMD is [6]

$$
\mathrm{R}_{\mathrm{BMD}}=\max \left(0, \mathrm{H}(X)-\sum_{k=1}^{m} \mathrm{H}\left(B_{k} \mid Y\right)\right) .
$$

For symbol-wise HD, one can achieve

$$
\mathrm{R}_{\mathrm{HD}, \mathrm{SMD}}=\max \left(0, \mathrm{H}(X)-\left(\mathrm{H}_{2}(\delta)+\delta \log _{2}(M-1)\right)\right)
$$




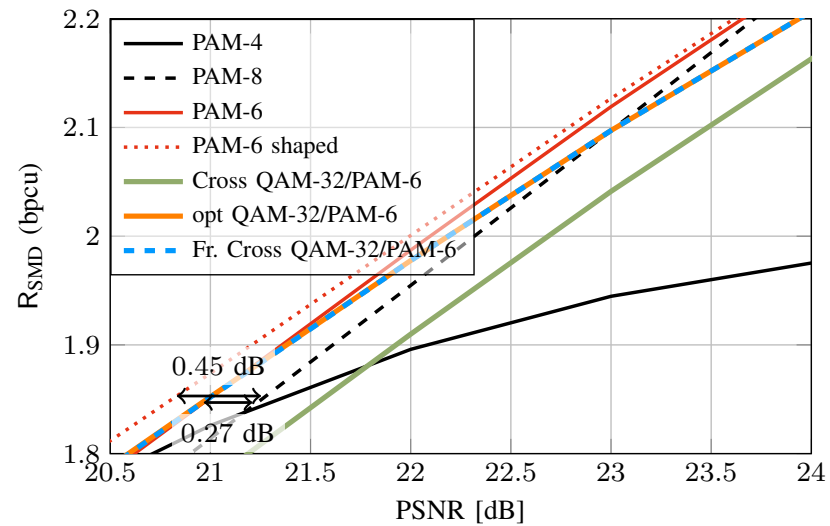

(a) SEs with soft SMD.

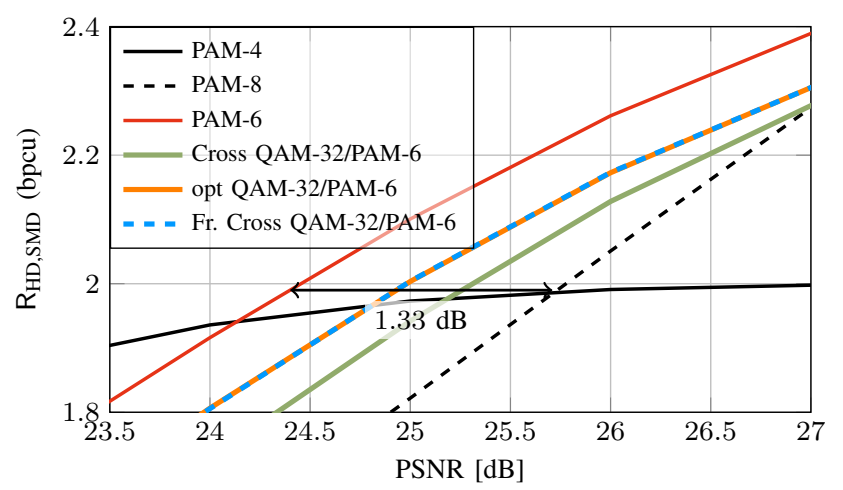

(c) SEs with SMD using hard decisions.

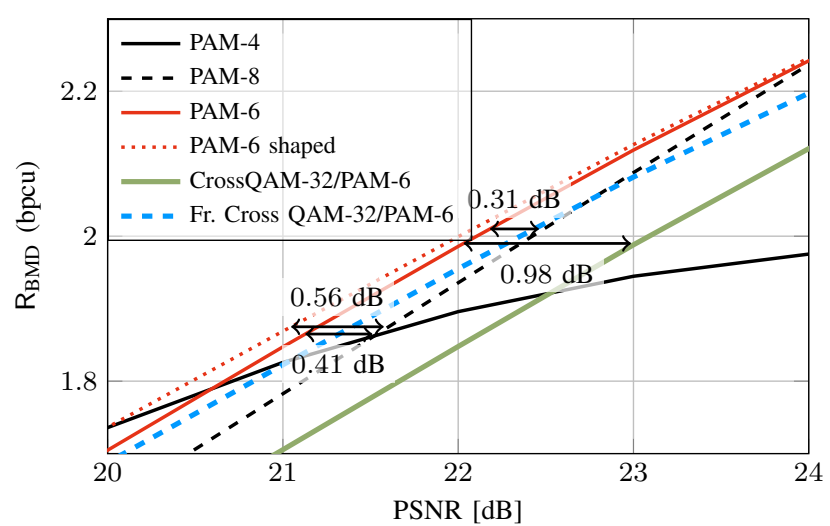

(b) SEs with soft BMD.

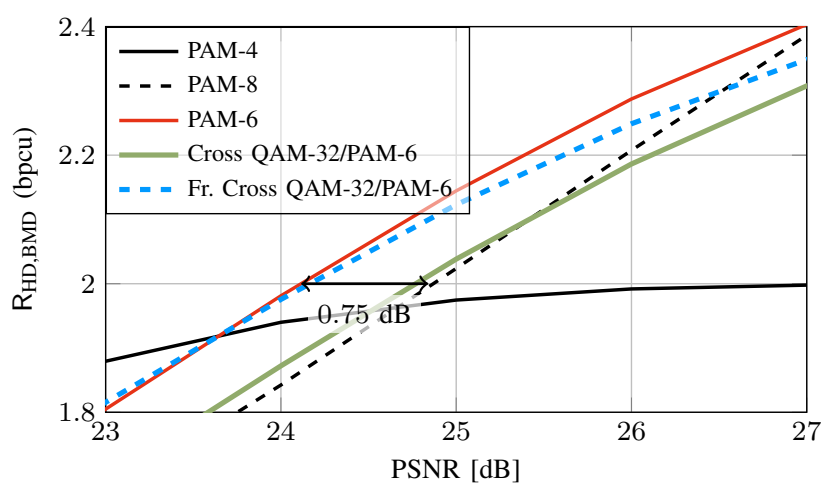

(d) SEs with BMD using hard decisions.

Fig. 1. SEs of PAM- $M$ with $M=4,6,8$ and QAM-32 for the peak-power constrained AWGN channel.

where $\mathrm{H}_{2}(\cdot)$ is the binary entropy function and $\delta=\operatorname{Pr}[\hat{X} \neq X]$ is the HD symbol error probability with $\hat{X}$ being the symbolwise hard decisions fed into the detector.

With BMD the SE under HD is

$$
R_{\mathrm{HD}, \mathrm{BMD}}=\max \left(0, \mathrm{H}(X)-m \mathrm{H}_{2}(\varepsilon)\right)
$$

where $\varepsilon=1 / m \cdot \sum_{k=1}^{m} \operatorname{Pr}\left[\hat{B}_{k} \neq B_{k}\right]$ is the average harddecision bit error probability with $\hat{B}_{k}$ being the bit-wise hard decision of the $k^{\text {th }}$ bit fed into the detector.

Fig. 1 shows the SEs for SD and HD using SMD and BMD, respectively, for PAM- $M$ with $M=4,6,8$ and for PAM-6 based on two-dimensional QAM-32 constellations [4], [5]. The PAM- $M$ curves are for uniformly-spaced PAM constellations as defined in (2). PAM-6 using 32-QAM constellations is introduced and discussed in Sec. [II] In Sec. IV] we explain how PAM-6 can be implemented using PAS.

The PAM- $M$ curves in Fig. 11 show that each decoding method has a PSNR region where PAM-6 outperforms PAM-4 and PAM-8. For SD-SMD (Fig. 1a), PAM-6 gains up to $0.27 \mathrm{~dB}$ and $0.45 \mathrm{~dB}$ PSNR compared to PAM- 8 by using a uniform and optimized input distribution (dotted red), respectively. For a rate of 1.85 bits/per channel use (bpcu), the optimized distribution for SD-SMD is shown in Fig. 2. Under SD-BMD (Fig. 1b) PAM-6 gains up to $0.41 \mathrm{~dB}$ and $0.56 \mathrm{~dB}$ PSNR compared to PAM- 8 by using a uniform and optimized

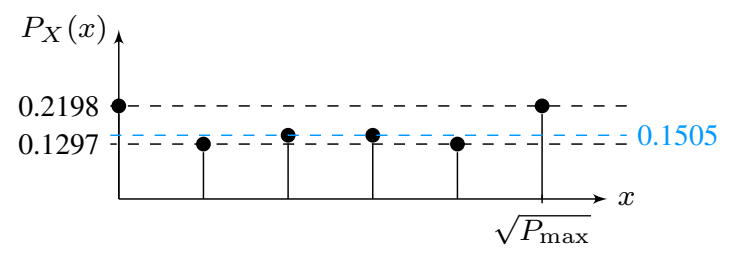

Fig. 2. Optimal distribution $P_{X}(x)$ of PAM-6 for SD-SMD at a rate of 1.85 bpcu.

input distribution, respectively. For $\mathrm{HD}$, the gain is up to $1.33 \mathrm{~dB}$ and $0.75 \mathrm{~dB}$ PSNR with SMD (Fig. 1c) and BMD (Fig. 1d), respectively.

\section{6-PAM USING QAM-32 CONSTELLATIONS}

\section{A. Cross QAM-32 constellation}

One-dimensional PAM-6 symbols can be created from a two-dimensional cross QAM-32 constellation [4], [5] as depicted in Fig. 3. QAM-32 is a simple choice for PAM-6 since 5 bits are directly mapped to the QAM-32 symbols. We use a quasi symmetric-ultracomposite (SU) labeling structure as introduced in [9]. The two-dimensional mapping based on QAM-32 is often referred to as PAM-6 in the literature, whereas by PAM- 6 we mean a one-dimensional constellation with 6 uniformly spaced points according to 22. Fig. 1 shows 


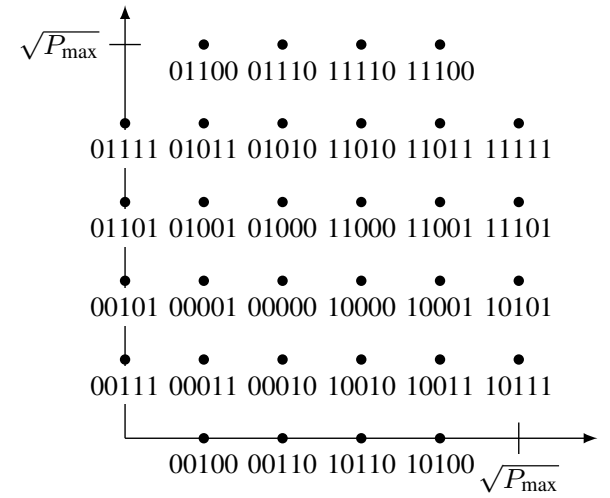

Fig. 3. Cross QAM-32 constellation with quasi-SU labeling [9].

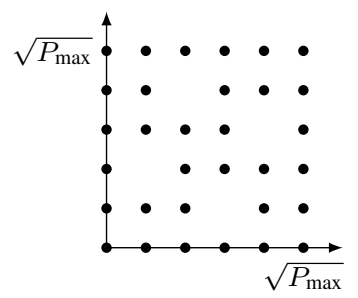

Fig. 4. Optimal QAM-32 constellation for SMD under a peak power constraint.

that cross QAM-32 exhibits a large loss in PSNR compared to PAM-6 under a peak power constraint. Note that we calculate the rates (4)-(7) with the complex-valued 32-QAM constellation and divide the resulting rate by two in order to obtain the rate in bits per real channel use.

\section{B. Framed-cross QAM-32 constellation}

Fig. 2 shows that the optimal input distribution under a peak power constraint has a higher probability at the outer constellation points. But this is the opposite as the cross QAM-32 constellation where the inner constellation points are used more often than the outer ones. We thus formulate an optimization problem to obtain the QAM-32 constellation that achieves the highest rates.

Define the QAM-36 constellation

$$
\mathcal{X}_{36}=\{a+\mathrm{j} b \mid a, b \in \mathcal{X}\}
$$

where we use $\mathcal{X}$ from (2) with $M=6$. Now maximize the SMD-SD rate over all 32-symbol subsets $\tilde{\mathcal{X}}$ of $\mathcal{X}_{36}$ :

$$
\mathcal{X}_{32, \mathrm{SMD}}^{\star}=\underset{\substack{\tilde{\mathcal{X}} \subset \mathcal{X}_{36} \\|\tilde{\mathcal{X}}|=32}}{\operatorname{argmax}} \mathrm{R}_{\mathrm{SMD}}
$$

The optimal constellation $\mathcal{X}_{32, \mathrm{SMD}}^{\star}$ according to 9 is depicted in Fig. 4. This constellation does not allow Gray labeling and finding the best bit labeling under BMD seems difficult. Instead, we modify (9) by taking the BMD rate from (5) as the new objective function. Furthermore, we impose a Gray labeling constraint were the first two bits of the label correspond to the quadrant of the constellation. The result is shown in Fig. 5 and we denote this constellation as framedcross QAM-32.

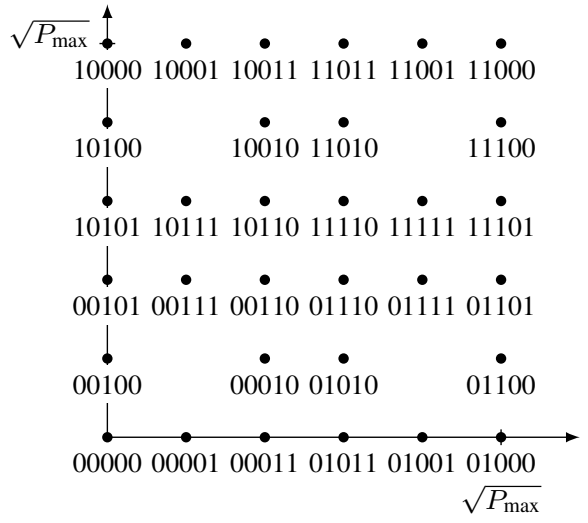

Fig. 5. Framed-cross QAM-32 constellation with Gray labeling.

The information rates of the optimized QAM-32 and framed-cross QAM-32 constellations are shown in Fig. 1] Fig. 1a and 1c show that there is no visible PSNR loss between the suboptimal framed-cross constellation and optimized QAM-32. Furthermore, framed-cross QAM-32 always outperforms cross QAM-32. For SD-SMD and HD-BMD, framed-cross QAM-32 achieves similar rates as PAM-6 if the rates are low. For HD-SMD and SD-BMD, the rate of framedcross QAM-32 lies between that of PAM-6 and cross QAM-32.

\section{IV. $M$-PAM USING PAS}

PAS was designed for distributions $P_{X}$ that are symmetric around zero [6]. However, PAS can be extended to any distribution with a symmetry. We propose a PAS scheme for PAM- $M$ distributions for even integers $M>2$. The number of bits needed to label the constellation points is $m=\left\lceil\log _{2} M\right\rceil$. The same idea is used in [10] to construct flexible QAM constellations.

The PAS scheme is illustrated in Fig. 6. A source $P_{D}$ outputs uniformly distributed data bits $d_{i}, i=1,2, \ldots, k+\gamma n$. A subsequence $d_{1}, \ldots, d_{k}$ is matched to a sequence $a_{1}, a_{2}, \ldots, a_{n}$ with a desired distribution $P_{A}($.$) by a distribu-$ tion matcher (DM). We use constant composition distribution matching (CCDM) [11] in this paper, but other DMs can be used as well. The "amplitude" constellation $\mathcal{A}$ is the set of the first $M / 2$ symbols of the PAM- $M$ constellation $\mathcal{X}$ :

$$
\mathcal{A}=\left\{i \cdot \frac{\sqrt{P_{\max }}}{M-1}\right\}_{i=0}^{M / 2-1} .
$$

Every point $a \in \mathcal{A}$ has a binary label $\boldsymbol{b}(a)=\left(b_{2}, \ldots, b_{m}\right)$ of length $m-1$. We use subsets of binary reflected Gray codes (BRGC) [12] of length $m-1$, where we discard the first $2^{m-1}-M / 2$ codewords from the usual Gray code (compare the labeling of $A=B_{2} B_{3}$ in Fig. 77.

The FEC code $\mathcal{C}$ is binary, and has length $m \cdot n$ and dimension $(m-1+\gamma) n$ with $0 \leq \gamma \leq 1$. Let $\boldsymbol{P}$ be the parity-generating matrix of a systematic generator matrix $\boldsymbol{G}=\left[\boldsymbol{I}_{k} \mid \boldsymbol{P}\right]$. Systematic encoding multiplies the bit string of length $(m-1+\gamma) n$ with $\boldsymbol{P}$, where the bit string consists of the binary labels and $\gamma n$ additional uniformly distributed bits 


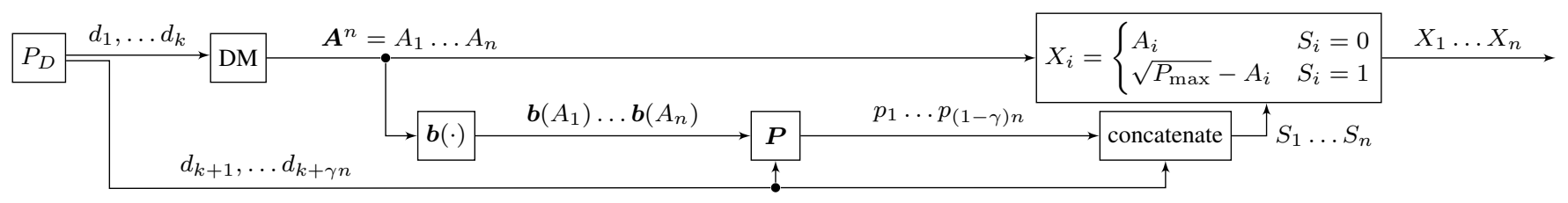

Fig. 6. Block diagram for probabilistic amplitude shaping (PAS).

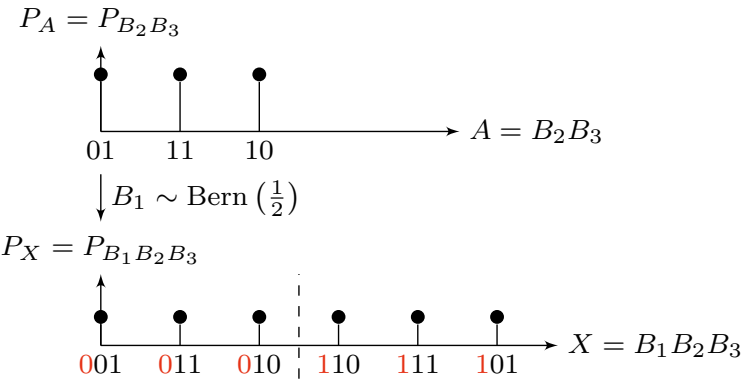

Fig. 7. Generating PAM-6 symbols using PAS: PAM-3 symbols are generated using a DM and labelled with two bits. Uniform parity bits are used to extend the PAM-3 symbols to PAM-6 symbols with a three bit label.

$d_{k+1}, \ldots, d_{k+\gamma n}$ from the source. This results in $(1-\gamma) n$ parity bits $p_{1}, \ldots, p_{(1-\gamma) n}$ that we assume to be uniformly distributed at the decoder [13]. Together with the $\gamma n$ bits $d_{k+1}, \ldots, d_{k+\gamma n}$, we have $n$ bits that map the $M / 2$ symbols of $\mathcal{A}$ to either $\mathcal{A}$ or $\mathcal{X} \backslash \mathcal{A}$.

The SE of this scheme is given by (4)-(7). The overall SE including DM and FEC is

$$
\mathrm{SE}=R_{\mathrm{DM}}+1-m\left(1-R_{\mathrm{FEC}}\right)
$$

where $R_{\mathrm{DM}}$ denotes the rate of the $\mathrm{DM}$ and $R_{\mathrm{FEC}}$ the code rate of the FEC code. The relation to $\mathrm{H}(X)$ in (4)-(7) is

$$
R_{\mathrm{DM}}+1 \leq \mathrm{H}(A)+1=\mathrm{H}(X)
$$

where $\mathrm{H}(A) \leq \log _{2}(M)-1$, with equality iff $A$ is uniformly distributed. The FEC overhead $m\left(1-R_{\mathrm{FEC}}\right)$ is larger or equal to the terms that are substracted from $\mathrm{H}(X)$ in (4)(7). The difference between $R_{\mathrm{DM}}$ and $\mathrm{H}(A)$ is called the rate loss, and it approaches zero asymptotically in the DM output length [11].

\section{COMPARISON / NumeriCAL RESUlTS}

We evaluate PAM-6 with PAS and compare it to PAM-6 generated by $32-\mathrm{QAM}$, and to PAM- 8 . We further compare SEs for both HD and SD and provide coded results employing low-density parity-check (LDPC) codes. We perform 200 and 20 belief-propagation iterations with a full sum-product update rule for $\mathrm{SD}$ and $\mathrm{HD}$, respectively.

\section{A. SD-BMD using $5 G$ LDPC codes}

Compared to SMD, BMD with soft-decoding exhibits a loss in PSNR, as BMD uses a mismatched decoding metric instead of $P_{X \mid Y}$. The PSNR loss for PAM-6, PAM-8 and QAM-32/PAM is shown in Fig. 8 as the gap between the dashed (BMD) and solid (SMD) curves. The PAM-6 loss is

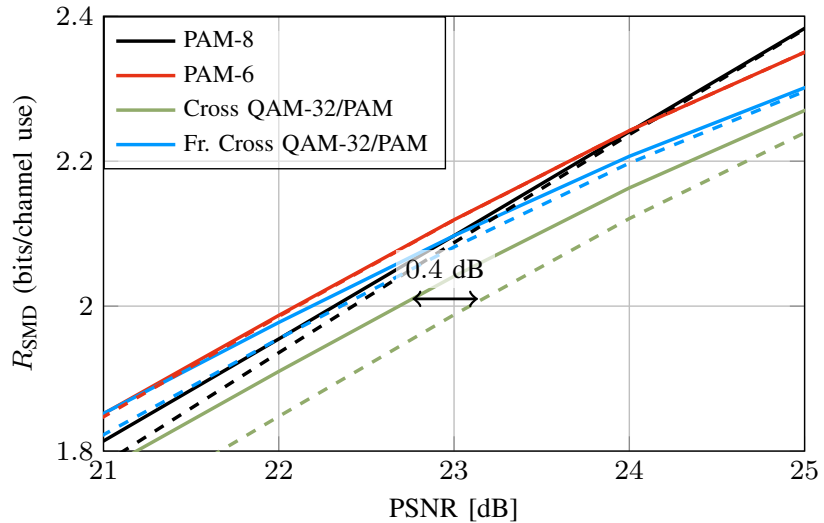

Fig. 8. SEs under BMD (dashed) and SMD (solid) soft-decoding.

negligible in the PSNR region of interest, whereas a $0.4 \mathrm{~dB}$ loss between SMD and BMS is evident for cross QAM-32.

In the following, we compare the schemes at a rate of 2 bpcu. As shown in Fig. 1b, PAM-6 gains $0.31 \mathrm{~dB}$ over PAM- 8 and $0.98 \mathrm{~dB}$ over cross QAM-32. PAM-6 outperforms PAM-8 up to a SE of $R=2.25 \mathrm{bpcu}$. The cross QAM-32 SE is lower than PAM-6 and PAM-8 over the entire PSNR range.

The benefits of PAS-PAM-6 and framed-cross QAM-32 over cross QAM-32 are twofold:

1) The uniform distribution of PAS-PAM- 6 and the nonuniform distribution implied by framed-cross QAM-32 are closer to the optimal distribution (cf. Fig. 2) under a peak-power constraint than the distribution implied by cross QAM-32.

2) The BMD loss is minimized due to the "true" Gray labeling of PAS-PAM-6 and framed-cross QAM-32.

PAS-PAM-6 has an additional small gain: the entropy of PAM-6 is $\log _{2}(6) \approx 2.58$ bpcu compared to 2.5 bpcu for the QAM-32 constellations.

The SEs give reasonable predictions of the PSNR gains with implemented codes, as shown by the frame error rate (FER) curves in Fig. 9. We use LDPC codes as described in the 5G new radio standard [14] with a block length of $n=3000$ channel uses (3000 PAM-6/8 symbols and 1500 QAM-32 symbols, respectively). The overall SE is $2 \mathrm{bpcu}$. The code parameters are summarized in Table II. The rate loss of the CCDM is 0.004 bpcu. At a FER of $10^{-3}$, PAM- 6 using PAS gains $0.4 \mathrm{~dB}$ and $0.84 \mathrm{~dB}$ compared to PAM- 8 and cross QAM-32, respectively. The FER of framed-cross QAM-32 is within $0.13 \mathrm{~dB}$ of PAM-6. These results are in line with the SEs from Fig. $1 \mathrm{~b}$. 


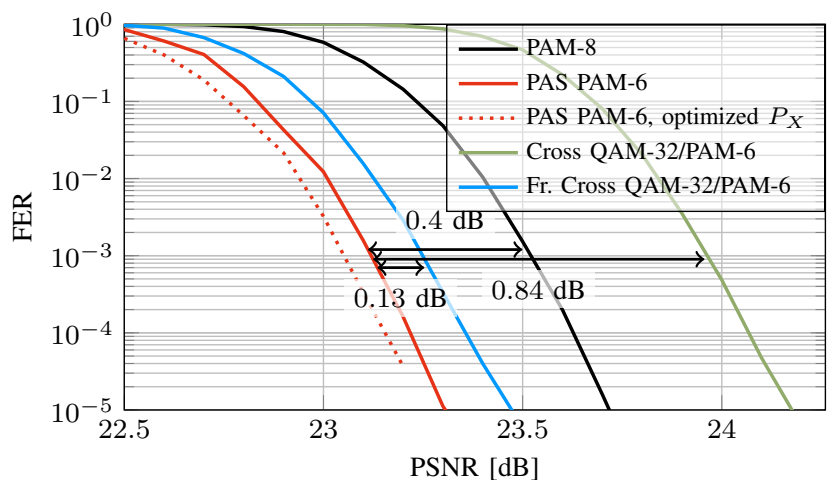

Fig. 9. Performance comparison of PAM-6 schemes at a SE of $2 \mathrm{bpcu}$ under SD-BMD with $n=3000$ channel uses per frame.

\begin{tabular}{||r|l|l|l||}
\hline Scenario & code length & coderate $R_{\text {FEC }}$ & $\gamma n$ \\
\hline \hline PAS PAM-6 & 9000 bits & $7257 / 9000 \approx 0.8$ & 1257 bits \\
PAM-8 & 9000 bits & $2 / 3$ & \\
QAM-32/PAM-6 & 7500 bits & 0.8 & \\
\hline
\end{tabular}

TABLE I

CODE PARAMETERs.

\section{B. HD using $5 G$ LDPC Codes}

SD FEC codes may be too complex for high-throughput applications such as IM/DD for short-reach fiber-optic links. Instead, one often uses HD. SEs for HD are depicted in Fig. 1. Observe that BMD can improve on SMD under HD, see Figs. $1 \mathrm{c}$ and $1 \mathrm{~d}$, see [15]. The reason is that BMD works with the binary Hamming distance that preserves part of the Euclidean distance, while the $M$-ary Hamming metric for SMD does not. To validate the results, Fig. 10 shows the performance of 5G LDPC codes with HD. We make bit-wise hard decisions on the channel outputs and assign the values $+a$ and $-a$ to the LLRs if the decision is 0 or 1 , respectively. The value $a$ is the same for all bit-levels and symbols and the optimal value of $a$ is found with a line search. We use the same simulation parameters as for SD decoding, i.e., $n=3000$ channel uses and a target SE of 2 bpcu. PAS-PAM- 6 gains $0.83 \mathrm{~dB}$ and $0.65 \mathrm{~dB}$ over PAM- 8 and QAM-32 based PAM- 6 , respectively. This confirms the SE results from Fig. 1d.

We remark that there are codes that perform better under HD-BMD than 5G LDPC codes. The aim here is to show that information rate gains predict the real coding gains, and $5 \mathrm{G}$ LDPC codes allow a good rate adaption to approach the rates described in Tab. []

\section{CONCLUSiOnS}

PAM-6 exhibits PSNR gains compared to PAM-4 and PAM-8 when transmitting over an AWGN channel with a peak power constraint. We proposed a PAS scheme to generate PAM-6 symbols and show that this schemes outperforms PAM-8 and a conventional PAM-6 scheme that is based on a 2-D to 1-D mapping of a complex-valued cross-shaped QAM-32 constellation. Additionally, we introduced an optimized QAM-32 constellation with the shape of a framed cross that achieves similar PSNR gains as the previously mentioned PAS scheme. The binary code length of framed-cross QAM-32

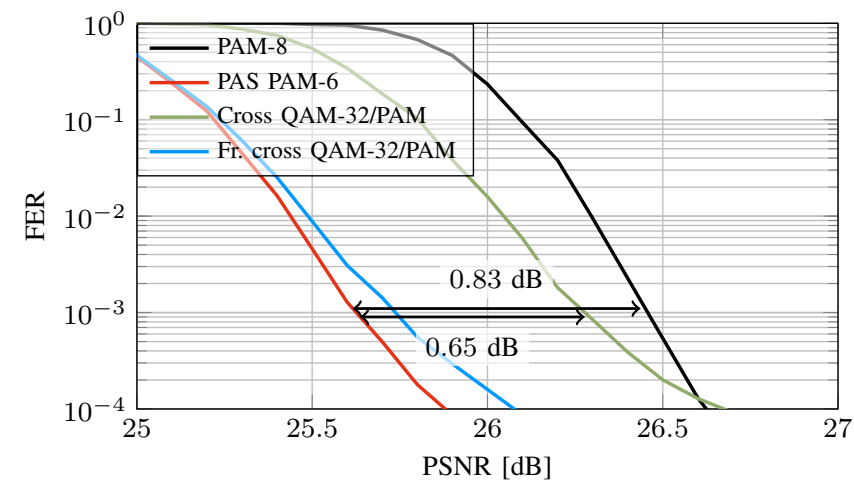

Fig. 10. Performance of PAS-PAM-6 at a SE of 2 bpcu under HD-BMD using 5G LDPC codes with $n=3000$ channel uses per frame.

is less than for the PAS scheme, which might be beneficial in cheap hardware. The additional gains of the PAS scheme do come at the cost of increasing computational complexity due to the DM. Using the DM to optimize the input distribution achieves further PSNR gains.

\section{REFERENCES}

[1] M. Chagnon, "Optical communications for short reach," J. Lightw. Technol., vol. 37, no. 8, pp. 1779-1797, 2019.

[2] K. Zhong, X. Zhou, J. Huo, C. Yu, C. Lu, and A. P. T. Lau, "Digital signal processing for short-reach optical communications: A review of current technologies and future trends," J. Lightw. Technol., vol. 36, no. 2, pp. 377-400, 2018.

[3] T. Wiegart, F. Da Ros, M. P. Yankov, F. R. Steiner, S. Gaiarin, and R. D. Wesel, "Probabilistically shaped 4-PAM for short-reach IM/DD links with a peak power constraint," J. Lightw. Technol., 2020.

[4] A. Ghiasi, Z. Wang, and V. Telang, "Investigation of PAM-4/6/8 signaling and FEC for $100 \mathrm{~Gb} / \mathrm{s}$ serial transmission," IEEE 802.3bm Task Force, 2012.

[5] L. Chorchos, "PAM-6 generation using 32-QAM constellation," in Metro and Data Center Optical Networks and Short-Reach Links II, vol. 10946. International Society for Optics and Photonics, 2019.

[6] G. Böcherer, F. Steiner, and P. Schulte, "Bandwidth efficient and rate-matched low-density parity-check coded modulation," IEEE Trans. Commun., vol. 63, no. 12, pp. 4651-4665, 2015.

[7] G. Böcherer, P. Schulte, and F. Steiner, "Probabilistic shaping and forward error correction for fiber-optic communication systems," $J$. Lightw. Technol., vol. 37, no. 2, pp. 230-244, Jan. 2019.

[8] G. Böcherer, Principles of Coded Modulation. Technische Universität München, 2018. [Online]. Available: http://www.georg-boecherer.de/ bocherer2018principles.pdf

[9] R. D. Wesel, X. Liu, J. M. Cioffi, and C. Komninakis, "Constellation labeling for linear encoders," IEEE Trans. on Inf. Theory, vol. 47, no. 6, pp. 2417-2431, 2001

[10] F. Buchali, Q. Hu, M. Chagnon, K. Schuh, L. Schmalen, and S. Makovejs, "Flexible transmission enabled by novel m2-qam formats with record distance-spectral efficiency tuneability," in Optical Fiber Communication Conference. Optical Society of America, 2018, pp. W2A-54.

[11] P. Schulte and G. Böcherer, "Constant composition distribution matching," IEEE Trans. Inf. Theory, vol. 62, no. 1, pp. 430-434, 2015.

[12] F. Gray, "Pulse code communication," Mar. 17 1953, US Patent $2,632,058$.

[13] G. Böcherer, "Labeling non-square QAM constellations for onedimensional bit-metric decoding," IEEE Commun. Lett., vol. 18, no. 9, pp. 1515-1518, 2014.

[14] "3GPP TS 38.212 V15.0.0: Multiplexing and channel coding," Dec. 2017.

[15] A. Sheikh, A. G. i Amat, and G. Liva, "Achievable information rates for coded modulation with hard decision decoding for coherent fiber-optic systems," J. Lightw. Technol., vol. 35, no. 23, pp. 5069-5078, 2017. 